\title{
Formation of methotrexate-PLLA-PEG-PLLA
}

\section{composite microspheres by microencapsulation through a process of suspension-enhanced dispersion by supercritical $\mathrm{CO}_{2}$}

\author{
This article was published in the following Dove Press journal: \\ International Journal of Nanomedicine \\ 16 June 2012 \\ Number of times this article has been viewed
}

\author{
Ai-Zheng Chen ${ }^{1,2}$ \\ Guang-Ya Wang' \\ Shi-Bin Wang ${ }^{1,2}$ \\ Li Li' \\ Yuan-Gang Liu',2 \\ Chen Zhao' \\ 'College of Chemical Engineering, \\ ${ }^{2}$ Institute of Pharmaceutical \\ Engineering, Institute of Biomaterials \\ and Tissue Engineering, Huaqiao \\ University, Xiamen 361021, China
}

\begin{abstract}
Background: The aim of this study was to improve the drug loading, encapsulation efficiency, and sustained-release properties of supercritical $\mathrm{CO}_{2}$-based drug-loaded polymer carriers via a process of suspension-enhanced dispersion by supercritical $\mathrm{CO}_{2}(\mathrm{SpEDS})$, which is an advanced version of solution-enhanced dispersion by supercritical $\mathrm{CO}_{2}$ (SEDS).

Methods: Methotrexate nanoparticles were successfully microencapsulated into poly (L-lactide)-poly(ethylene glycol)-poly(L-lactide) (PLLA-PEG-PLLA) by SpEDS. Methotrexate nanoparticles were first prepared by SEDS, then suspended in PLLA-PEG-PLLA solution, and finally microencapsulated into PLLA-PEG-PLLA via SpEDS, where an "injector" was utilized in the suspension delivery system.
\end{abstract}

Results: After microencapsulation, the composite methotrexate (MTX)-PLLA-PEG-PLLA microspheres obtained had a mean particle size of $545 \mathrm{~nm}$, drug loading of $13.7 \%$, and an encapsulation efficiency of $39.2 \%$. After an initial burst release, with around $65 \%$ of the total methotrexate being released in the first 3 hours, the MTX-PLLA-PEG-PLLA microspheres released methotrexate in a sustained manner, with $85 \%$ of the total methotrexate dose released within 23 hours and nearly $100 \%$ within 144 hours.

Conclusion: Compared with a parallel study of the coprecipitation process, microencapsulation using SpEDS offered greater potential to manufacture drug-loaded polymer microspheres for a drug delivery system.

Keywords: drug loading, encapsulation efficiency, methotrexate, nanoparticles, poly(L-lactide), supercritical $\mathrm{CO}_{2}$ sustained release

\section{Introduction}

Incorporating pharmaceutical compounds into a biodegradable polymer carrier is an effective strategy for forming a drug delivery system and has been given increasing attention. ${ }^{1-5}$ Yin and Yates reported that drug-entrapped biodegradable microcapsules, with sustained drug release, were prepared by spraying a suspension of hollow poly(DL-lactide) particles in procaine hydrochloride aqueous solution into plasticizing solvents. ${ }^{6}$ A further report by Zvonar et al described use of a self-microemulsifying system to fabricate $\mathrm{Ca}$-pectinate microcapsules to improve the release and permeability of furosemide. ${ }^{7}$

Drug-loaded polymer microspheres have been variously prepared by phase separation, ${ }^{8}$ interfacial polymerization, ${ }^{9}$ spray-drying, ${ }^{10}$ freeze-drying, ${ }^{11}$ and emulsion evaporation. ${ }^{12}$ However, these conventional strategies always either involve an 
intense operating process or preparation of particles with a wide particle size distribution and a relatively large amount of organic solvent residue. Compared with these traditional methodologies, supercritical $\mathrm{CO}_{2}$-based technology offers a number of advantages, including mild critical conditions ( $T c=304.1 \mathrm{~K}, P c=7.38 \mathrm{MPa})$, no toxicity, a nonorganic residue, are not flammable, and all at a relatively lower price. $^{13-15}$

The supercritical antisolvent process has been widely utilized to fabricate drug-loaded polymer microparticles. ${ }^{16-18}$ In the supercritical antisolvent process, an organic solution is sprayed into supercritical $\mathrm{CO}_{2}$ to effect immediate contact between the two media. Extremely rapid extraction of the organic solvent into supercritical $\mathrm{CO}_{2}$ leads to higher supersaturation of the solution, creating fast nucleation and growth, and finally micronization of the solute. Similarly, when the drug and polymer are both dissolved to obtain a homogeneous organic solution and sprayed into supercritical $\mathrm{CO}_{2}$, the drug and polymer are coprecipitated due to a higher supersaturation caused by diffusion of the organic solvent into the supercritical $\mathrm{CO}_{2}$ and vice versa, ${ }^{19,20}$ known as the "coprecipitation" process. However, the coprecipitation process generates drug-loaded polymer microspheres accompanied by relatively low drug loading, low encapsulation efficiency, and no sustained release of the drug, which can be partially explained by the fact that the drug is precipitated onto or just loosely attached to the surface of the polymer microspheres. ${ }^{19,21}$

To overcome the drawbacks of the coprecipitation process, ie, low drug loading and low encapsulation efficiency, the microencapsulation process was developed ${ }^{19}$ whereby nanoparticles of a drug are first prepared, suspended in an organic polymer solution, and then sprayed into supercritical $\mathrm{CO}_{2}$ using a high-performance liquid chromatography (HPLC) pump. Due to precipitation of the polymer from the sprayed suspension, drug nanoparticles are entrapped by the polymer with a matrix or core-shell structure. ${ }^{19,22}$

In order to strengthen mutual diffusion between the sprayed solution and the supercritical $\mathrm{CO}_{2}$, a solutionenhanced dispersion by supercritical fluid (SEDS) process was developed and has been widely used in supercritical fluid techniques. ${ }^{23}$ During this process, the substrate(s) solution and supercritical $\mathrm{CO}_{2}$ are copulverized through a coaxial nozzle, in which supercritical $\mathrm{CO}_{2}$ acts as an antisolvent and spraying enhancer. ${ }^{24-27}$ However, when SEDS is used in the microencapsulation process, ie, driving, delivering, and spraying the suspension into the highpressure reaction vessel, the delivery system, especially the one-way valve, is easily damaged or obstructed by the suspended particles.

To tackle this problem, a suspension-enhanced dispersion by supercritical fluid (SpEDS) process was developed in the present study based on SEDS. With SpEDS, supercritical $\mathrm{CO}_{2}$ and a suspension consisting of drug nanoparticles and polymer solution are simultaneously sprayed through a stainless steel coaxial nozzle, whereby spraying and dispersion of the suspension is enhanced by spraying of supercritical $\mathrm{CO}_{2}$. To avoid obstruction and guarantee smooth delivery of the suspension, a stainless steel cylindrical container equipped with a piston working like an injector was introduced and added into the previous SEDS apparatus.

This study attempted to prepare drug-loaded polymer microspheres using a supercritical microencapsulation process with improved drug loading, encapsulation efficiency, and sustained release, compared with the coprecipitation process. Methotrexate is an antifolate drug which is nearly insoluble in water, is effective in the treatment of a range of malignant and autoimmune diseases, ${ }^{28}$ and was selected as the drug model in this study. The drug was micronized by SEDS, suspended in a PLLA-PEG-PLLA solution containing dichloromethane, and microencapsulated by PLLA-PEG-PLLA via SpEDS. For the purpose of comparison, a parallel study involving the coprecipitation process was carried out. The products were characterized by scanning electron microscopy (SEM) and Fourier transform infrared spectroscopy (FTIR). Drug loading, encapsulation efficiency, and drug release profiles as well as release kinetics were also studied.

\section{Materials and methods Materials}

The PLLA-PEG-PLLA triblock polymer with a gross molecular weight of $77 \mathrm{kDa}$ and $8 \%(\mathrm{w} / \mathrm{w})$ PEG (6000 kDa) was obtained from Jianbaokaiyuan Biomaterials Co, Ltd (Jinan, China). Methotrexate was sourced from Surui Medicine Co, Ltd (Suzhou, China). Dichloromethane and dimethyl sulfoxide were purchased from Sinopharm Chemical Reagent Co, Ltd (Beijing, China). $\mathrm{CO}_{2}$ (purity 99.9\%) was provided by Rihong Industrial Development Co, Ltd (Xiamen, China). All other reagents were of analytical reagent grade. Chemicals were used as received without further purification.

\section{Preparation of methotrexate nanoparticles by SEDS}

Methotrexate nanoparticles were prepared according to a method used in our previous study. ${ }^{29}$ Specifically, methotrexate was first dissolved in dimethyl sulfoxide, and 
acetone was then added to obtain a homogeneous solution in which acetone acted as an organic nonsolvent for methotrexate and thus increased the saturation ratio and reduced its concentration. The final concentration of methotrexate was $0.5 \%(\mathrm{w} / \mathrm{v})$; the dimethyl sulfoxide to acetone ratio was 1:4(v/v).

The methotrexate nanoparticles were micronized by SEDS, whereby the pressure and temperature of $\mathrm{CO}_{2}$ were stabilized at the desired levels, the solution of methotrexate in dimethyl sulfoxide and acetone began being delivered by a HPLC pump to the coaxial nozzle and sprayed simultaneously with the supercritical $\mathrm{CO}_{2}$, and methotrexate nanoparticles were generated due to extremely rapid precipitation of methotrexate. In the preparation process, the operating pressure, temperature, flow rate of $\mathrm{CO}_{2}$, and flow rate of the methotrexate solution were stabilized at $12 \mathrm{MPa}$, $306 \mathrm{~K}, 2000$ standard liters per hour, and $1 \mathrm{~mL}$ per minute, respectively.

\section{Microencapsulation of methotrexate into PLLA-PEG-PLLA by SpEDS}

Figure 1 is a schematic diagram of the SpEDS apparatus for microencapsulating methotrexate into PLLA-PEG-PLLA. The apparatus comprised three main sections, ie, a $\mathrm{CO}_{2}$ delivery system, a particle suspension delivery system, and a high pressure reaction vessel, the volume of which was $500 \mathrm{~mL}$. An "injector" was used to avoid obstruction of the pump used for suspension delivery.

The SEDS apparatus has all the same components as those for SpEDS, except for the injector. The injector consists of three stainless steel components, ie, a cylinder (inside diameter $25.0 \mathrm{~mm}$, outside diameter $35.0 \mathrm{~mm}$, length $155.0 \mathrm{~mm}$ ), an inbuilt columnar piston (height $20.0 \mathrm{~mm}$ ) girdled by a rubber ring, and two nuts (outside diameter $50 \mathrm{~mm}$, insider diameter $35.0 \mathrm{~mm}$, depth $18 \mathrm{~mm}$ ), one of which is connected to the coaxial nozzle with a stainless conduit and the other with an HPLC pump. The stainless steel cylinder is divided into two chambers by the piston. For our experiment, the front chamber was filled with a suspension consisting of methotrexate nanoparticles and a solution of PLLA-PEG-PLLA containing dichloromethane, and the back chamber was filled with water. When the HPLC pump was turned on, the piston started to be driven by water from the HPLC pump, pushing the suspension smoothly forward through the stainless conduit to the stainless steel coaxial nozzle.

The suspension placed in the front chamber of the injector was obtained by suspending methotrexate nanoparticles in the PLLA-PEG-PLLA solution containing dichloromethane by ultrasonic dispersion, with a final methotrexate concentration

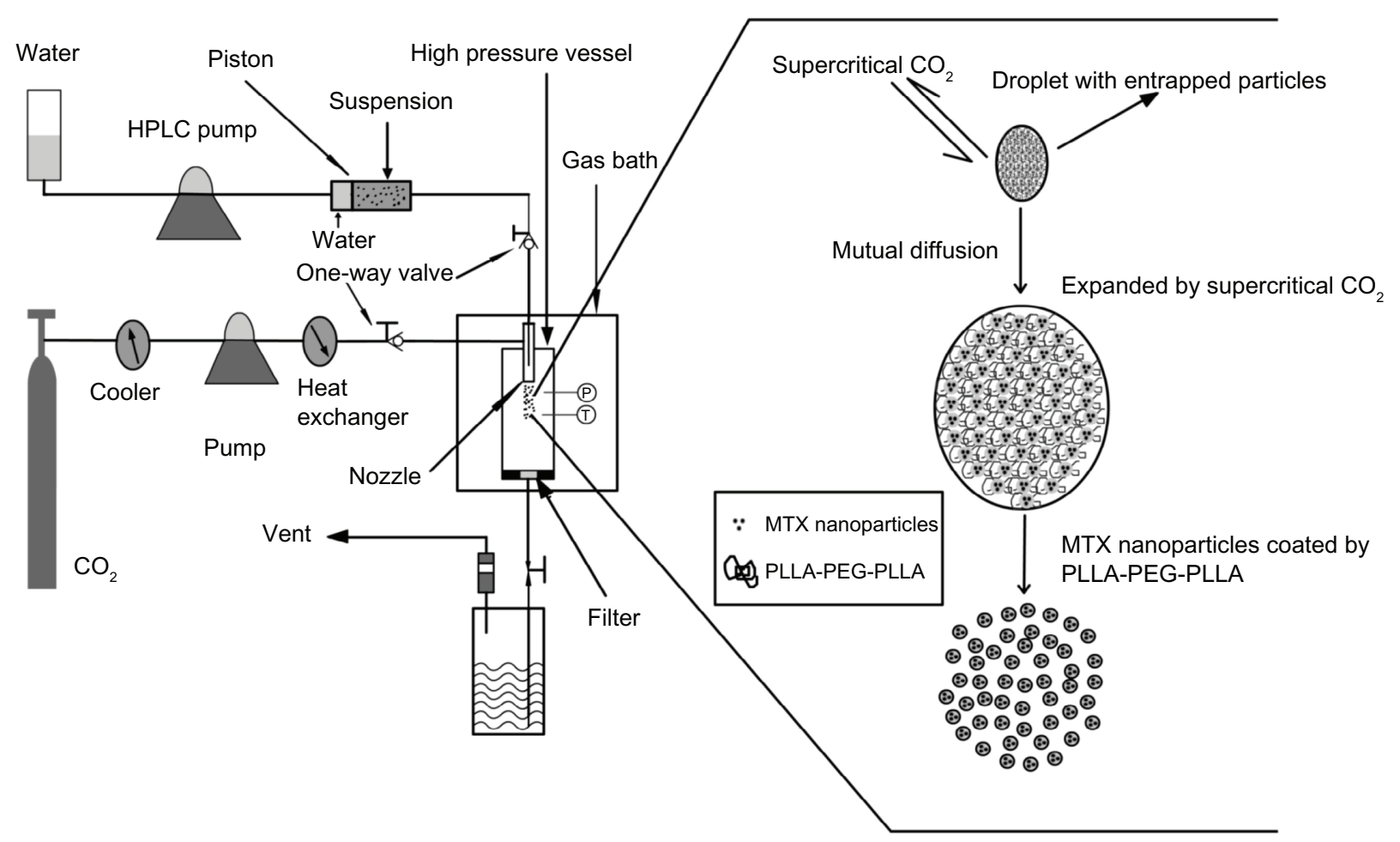

Figure I Schematic diagram of SpEDS apparatus and brief mechanism for the microencapsulation process.

Abbreviations: HPLC, high-performance liquid chromatography; MTX, methotrexate; SpEDS, suspension-enhanced dispersion by supercritical fluid. 
of $0.5 \%(\mathrm{w} / \mathrm{v})$ and a methotrexate to PLLA-PEG-PLLA ratio of $1: 9(\mathrm{w} / \mathrm{w})$.

In the microencapsulation experiment, the chosen pressure and temperature were first achieved and kept stable, and the methotrexate nanoparticle suspension was then driven by the designed injector and sprayed into the highpressure vessel through a stainless steel coaxial nozzle. The operating pressure, temperature, flow rate of $\mathrm{CO}_{2}$, and flow rate of the methotrexate solution were stabilized at $12 \mathrm{MPa}, 306 \mathrm{~K}, 2000$ standard liters per hour, and $1 \mathrm{~mL}$ per minute, respectively. After spraying on the suspension, fresh $\mathrm{CO}_{2}$ was continuously poured in, with the operating conditions unchanged, to wash the products for 30 minutes and completely remove the residual organic solvent. When washing was completed, the high-pressure vessel was slowly depressurized and the products were collected for subsequent analysis.

In order to make comparisons, a coprecipitation process involving methotrexate and PLLA-PEG-PLLA was carried out in parallel under the same operating conditions (pressure $12 \mathrm{MPa}$, temperature $306 \mathrm{~K}, \mathrm{CO}_{2}$ flow rate 2000 standard liters per hour, and a $1 \mathrm{~mL}$ per minute flow rate for the methotrexate solution). Briefly, methotrexate was first dissolved in dimethyl sulfoxide, and PLLA-PEG-PLLA solution containing dichloromethane was then added homogeneously, with a final PLLA concentration of $0.5 \%(\mathrm{w} / \mathrm{v})$, methotrexate to PLLA-PEG-PLLA ratios of 1:19, 1:9, and 3:17 (w/w), and a dimethyl sulfoxide to dichloromethane ratio of $1: 4(\mathrm{v} / \mathrm{v})$. Finally, the solution obtained was sprayed into supercritical $\mathrm{CO}_{2}$.

\section{Surface morphology and particle size distribution}

The surface morphology of the samples was investigated by SEM (Hitachi S-4800, Tokyo, Japan). Before observation, the sample was directly absorbed onto a piece of carbon film that was self-adhered on an aluminum stub. The particle size and particle size distribution were measured by analyzing the micrographs with Image-ProPlus 6.0 and Origin 8.5.

\section{FTIR analysis}

Sample powders and $\mathrm{KBr}$ were mixed gently to prepare $\mathrm{KBr}$ pellets. FTIR spectra of the original methotrexate and PLLA-PEG-PLLA, and the methotrexate (MTX)-PLLAPEG-PLLA microspheres prepared by the microencapsulation and coprecipitation processes were performed using an FTIR spectrometer (FTIR 8400S, Hitachi) in transmission mode, with a wavenumber range of $4000-400 \mathrm{~cm}^{-1}$.

\section{Drug loading and encapsulation efficiency}

Drug loading and encapsulation efficiency are both significant factors when evaluating the properties of drug-loaded polymer microspheres. To determine drug loading, $10 \mathrm{mg}$ of MTX-PLLA-PEG-PLLA microspheres was dissolved in $5 \mathrm{~mL}$ of dichloromethane; $30 \mathrm{~mL}$ of phosphate-buffered solution buffer ( $\mathrm{pH}$ 7.4) was added, and the solution was gently heated with magnetic stirring to evaporate the dichloromethane. The solution obtained was filtered through a $0.22 \mu \mathrm{m}$ membrane, and the concentration of methotrexate was measured using an ultraviolet spectrophotometer (Unic UV-2800, Culver City, CA) at $302.8 \mathrm{~nm}$ and comparing this with a standard concentration curve. For calculating the encapsulation efficiency, another $10 \mathrm{mg}$ of MTX-PLLAPEG-PLLA microspheres was dispersed in $10 \mathrm{~mL}$ of a dilute aqueous solution of sodium hydroxide $(0.1 \mathrm{~mol} / \mathrm{L})$ to wash off the unencapsulated and loosely surface-bound methotrexate; the resulting suspension was filtered through a $0.22 \mu \mathrm{m}$ membrane and the amount of methotrexate was determined using the ultraviolet spectrophotometer at $302.8 \mathrm{~nm}$ and calculating according to the standard concentration curve. Drug loading and encapsulation efficiency were calculated using the following two equations:

$$
\begin{gathered}
\text { Drug loading }=\mathrm{W}_{1} / \mathrm{W}_{2} \times 100 \% \\
\text { Encapsulation efficiency }=\mathrm{W}_{3} / \mathrm{W}_{1} \times 100 \%
\end{gathered}
$$

where $\mathrm{W}_{1}, \mathrm{~W}_{2}$, and $\mathrm{W}_{3}$ represent the weight of methotrexate in the microspheres, the gross weight of the microspheres, and the weight of methotrexate in the microspheres with the unencapsulated methotrexate washed off, respectively. Each experiment was performed in triplicate.

\section{Drug release studies}

To simulate and monitor the sustained release of methotrexate in the microspheres, release studies were carried out as follows. First, $10 \mathrm{mg}$ of MTX-PLLA-PEG-PLLA microspheres was placed in a pretreated dialysis bag. The bag was tied tightly, inserted in a sealed bottle containing $50 \mathrm{~mL}$ of phosphate-buffered solution ( $\mathrm{pH} 7.4)$, and incubated in a shaking waterbath at $310 \mathrm{~K}$ and $60 \mathrm{rpm}$. At preset time points (hours $0.5,1,2,3$ onwards), $5 \mathrm{~mL}$ of the incubated solution was periodically removed, with subsequent addition of $5 \mathrm{~mL}$ of fresh phosphate-buffered solution ( $\mathrm{pH}$ 7.4). The concentration of methotrexate in the aqueous solution was measured using the ultraviolet spectrophotometer at $302.8 \mathrm{~nm}$ and calculating according to the standard concentration curve. 
The sustained-release profiles of methotrexate were calculated in terms of the cumulative release percentage of methotrexate $(\mathrm{w} / \mathrm{w})$ with incubation time. Each experiment was carried out in triplicate.

\section{Results and discussion}

\section{Preparation of methotrexate nanoparticles}

Using the optimized operating parameters, together with addition of acetone as a nonsolvent to enhance the degree of saturation and reduce the methotrexate concentration in the initial solution, nanoparticles of methotrexate were successfully fabricated by SEDS. The surface morphology seen on SEM is shown in Figure 2, in which methotrexate nanoparticles can be observed to have a smooth surface. When observed by the human eye, the methotrexate nanoparticles had a fluffy and soft appearance resembling cotton-candy. This macroscopic surface morphology can be explained by the fact that methotrexate when processed by SEDS exists not in the form of connected aggregates but as free nanoparticles. ${ }^{30}$ Furthermore, methotrexate nanoparticles prepared by SEDS were shown to have a spherical shape with a relatively narrow particle size distribution, ie, a span of 0.73 and an average size of $86 \mathrm{~nm}$, as shown in Table 1.

\section{Surface morphology and particle size of MTX-PLLA-PEG-PLLA}

During the microencapsulation process, a suspension consisting of methotrexate nanoparticles and PLLA-PEG-PLLA in dichloromethane solution was driven by an injector and sprayed into a high-pressure vessel through a coaxial nozzle, where the jet stream of supercritical $\mathrm{CO}_{2}$ strengthened spraying of the suspension. When the droplets of suspension

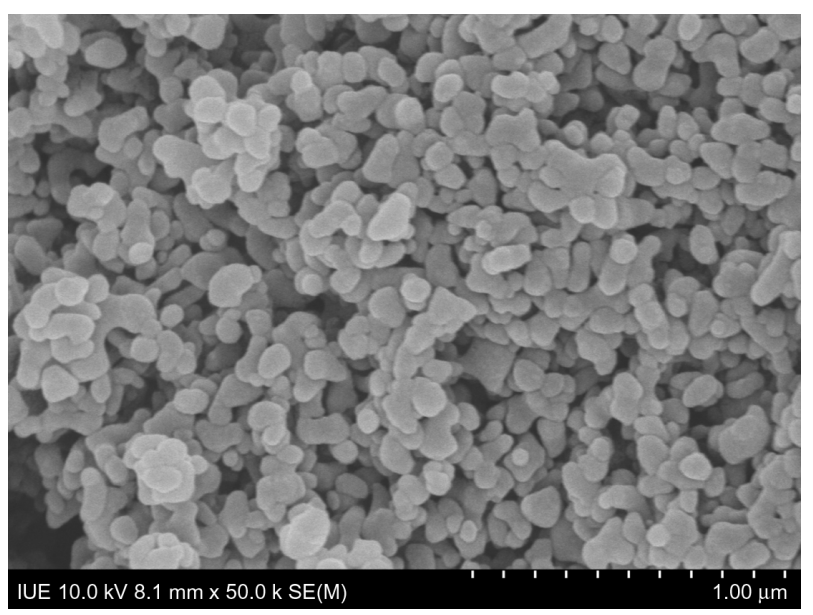

Figure 2 Scanning electron micrograph of methotrexate nanoparticles prepared by the solution-enhanced dispersion by supercritical fluid process.
Table I Particle size and particle size distribution of (A) methotrexate nanoparticles prepared by SEDS, and MTX-PLLAPEG-PLLA microspheres from (B) microencapsulation process and $(\mathbf{C})$ coprecipitation process with a drug dose of $10 \%$

\begin{tabular}{llllll}
\hline Samples & $D_{10}(\mathrm{~nm})$ & $\mathbf{D}_{50}(\mathrm{~nm})$ & $\mathbf{D}_{90}(\mathrm{~nm})$ & Span & $\begin{array}{l}\text { Mean size } \\
(\mathrm{nm})\end{array}$ \\
\hline A & 52 & 79 & 110 & 0.73 & 86 \\
B & 329 & 520 & 807 & 0.92 & 545 \\
C & 245 & 361 & 583 & 0.94 & 389 \\
\hline
\end{tabular}

Abbreviations: SEDS, solution-enhanced dispersion by supercritical fluid process; MTX-PLLA-PEG-PLLA, methotrexate-poly(L-lactide)-poly(ethylene glycol)-poly (L-lactide).

came into contact with supercritical $\mathrm{CO}_{2}$, a nonsolvent for PLLA-PEG-PLLA, extensive mutual diffusion into and out of the suspension droplets occurred due to the high solubility of dichloromethane in supercritical $\mathrm{CO}_{2}$. With regard to the PLLA-PEG-PLLA dichloromethane solution, the crystallization pathway shifted from the liquid-solution region into the solid-liquid region, via the liquid-solid and liquid-fluid two-phase region and the solid-liquid-fluid three-phase region, thus crossing a series of tie lines. When supersaturation of the PLLA-PEG-PLLA dichloromethane solution was approached, extraction of dichloromethane from the suspension droplet led to an extremely rapid phase change, with formation of a solid phase and precipitation of PLLA-PEG-PLLA onto the surface of the methotrexate nanoparticles. This encapsulation mechanism is briefly described in Figure 1.

Figure 3A and B illustrate the surface morphology of MTX-PLLA-PEG-PLLA microspheres manufactured by the microencapsulation process and the coprecipitation process, respectively. The corresponding particle size and particle size distribution are shown in Table 1. The particle size of the MTX-PLLA-PEG-PLLA microspheres, prepared by either the coprecipitation process or the microencapsulation process, appeared much larger than that of the methotrexate nanoparticles, indicating that most of the methotrexate nanoparticles were successfully encapsulated by PLLA-PEG-PLLA. The particle size of the MTX-PLLA-PEG-PLLA composite microspheres prepared by the microencapsulation process was somewhat larger than that of the composite microspheres generated by the coprecipitation process. This is because, in the microencapsulation process, not one but two or more methotrexate nanoparticles were encapsulated into PLLA-PEG-PLLA, generating relatively larger microspheres in which PLLA-PEG-PLLA acted as a matrix to entrap methotrexate nanoparticles, ie, the composite microspheres obtained in the microencapsulation process have an inlaymatrix structure rather than a typical core-shell structure. 

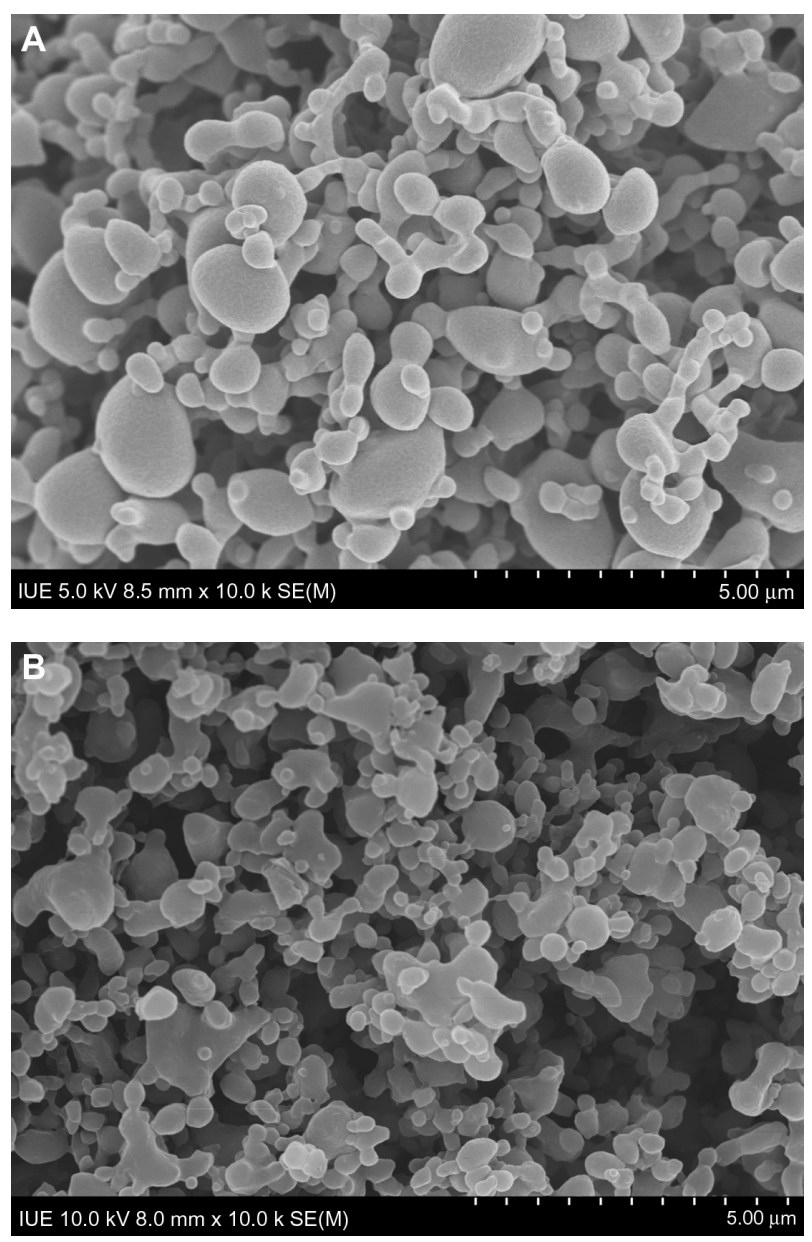

Figure 3 Scanning electron micrograph of MTX-PLLA-PEG-PLLA microspheres from (A) the microencapsulation process and (B) the coprecipitation process. Abbreviation: MTX-PLLA-PEG-PLLA, methotrexate-poly(L-lactide)-poly(ethylene glycol)-poly(L-lactide).

Judging from the span, MTX-PLLA-PEG-PLLA composite microspheres prepared by the microencapsulation and coprecipitation processes both had a narrow particle size distribution and there was no significant difference between them.

\section{FTIR analysis}

FTIR is a widely used and very useful tool in chemistry for analyzing chemical components and identifying characteristic groups. As shown in the FTIR spectra of Figure 4, there are three major regions of the FTIR spectra for PLLA-PEG-PLLA, ie, a $\mathrm{C}=\mathrm{O}$ stretching vibration region at $1820-1700 \mathrm{~cm}^{-1}$, a region at $1500-1000 \mathrm{~cm}^{-1}$ attributable to $\mathrm{CH}_{3}, \mathrm{CH}$ bending, and $\mathrm{C}-\mathrm{O}-\mathrm{C}$ stretching vibration, and a region at $980-820 \mathrm{~cm}^{-1}$ for $\mathrm{C}-\mathrm{C}$ backbone stretching vibration.

Among these three regions, the major peak at $1759 \mathrm{~cm}^{-1}$ is due to the stretching vibration of the carbonyl group in PLLA-PEG-PLLA and can be used to identify PLLAPEG-PLLA; this major peak can also be observed in the
MTX-PLLA-PEG-PLLA microspheres prepared by the two different processes. In the MTX-PLLA-PEG-PLLA microsphere spectrum, a weak peak of PLLA-PEG-PLLA at $1620 \mathrm{~cm}^{-1}$ was considerably enhanced due to the existence of characteristic peaks of methotrexate at $1718 \mathrm{~cm}^{-1}$ and $1604 \mathrm{~cm}^{-1}$, representing $\mathrm{C}=\mathrm{O}$ stretching vibration and $\mathrm{C}=\mathrm{C}$ benzene backbone stretching vibration, respectively. This result again confirms that methotrexate was encapsulated into or absorbed onto the microspheres.

Apart from that, no significant difference and no new peaks were found in the FTIR spectrum of the composite microspheres prepared by either the microencapsulation process or the coprecipitation process, ie, no chemical reaction occurred in the two kinds of process. This all demonstrates that SpEDS modified on the basis of SEDS is a typically physical process causing microencapsulation of drug nanoparticles via a biodegradable polymer, and that this process is favorable for drugs, because chemical interaction may alter the properties of drugs and reduce their effectiveness.

\section{Drug load and efficiency of encapsulation}

Figure 5 illustrates the drug loading and encapsulation efficiency of MTX-PLLA-PEG-PLLA microspheres prepared by the microencapsulation process and by the coprecipitation process. When the drug dose was set at $10 \%(\mathrm{w} / \mathrm{w})$, drug loading of MTX-PLLA-PEG-PLLA microspheres prepared by the microencapsulation process was $13.7 \% \pm 1.8 \%$, which was higher than the theoretical drug loading $(10 \%, \mathrm{w} / \mathrm{w})$, whereas drug loading $(8.3 \% \pm 2.4 \%)$ was lower than the theoretical one for the coprecipitation process. This phenomenon indicates that PLLA-PEG-PLLA was partially dissolved in supercritical $\mathrm{CO}_{2}$ and flushed out by running fluid in the microencapsulation process, and methotrexate nanoparticles were hardly lost. However, more methotrexate than PLLA-PEG-PLLA was lost during the coprecipitation process, which could be explained by the fact that precipitation of PLLA-PEG-PLLA was faster and easier than that of methotrexate.

Furthermore, the encapsulation efficiency of MTX-PLLAPEG-PLLA microspheres prepared by the microencapsulation process was $39.2 \% \pm 5.9 \%$, indicating that more methotrexate was encapsulated in the composite microspheres. A relatively lower encapsulation efficiency of $20.4 \% \pm 6.3 \%$ was obtained in the coprecipitation process, indicating that more methotrexate was precipitated and absorbed or combined loosely onto the surface of the composite microspheres.

Apart from that, when different drug doses were used in the coprecipitation process, drug loading increased and 


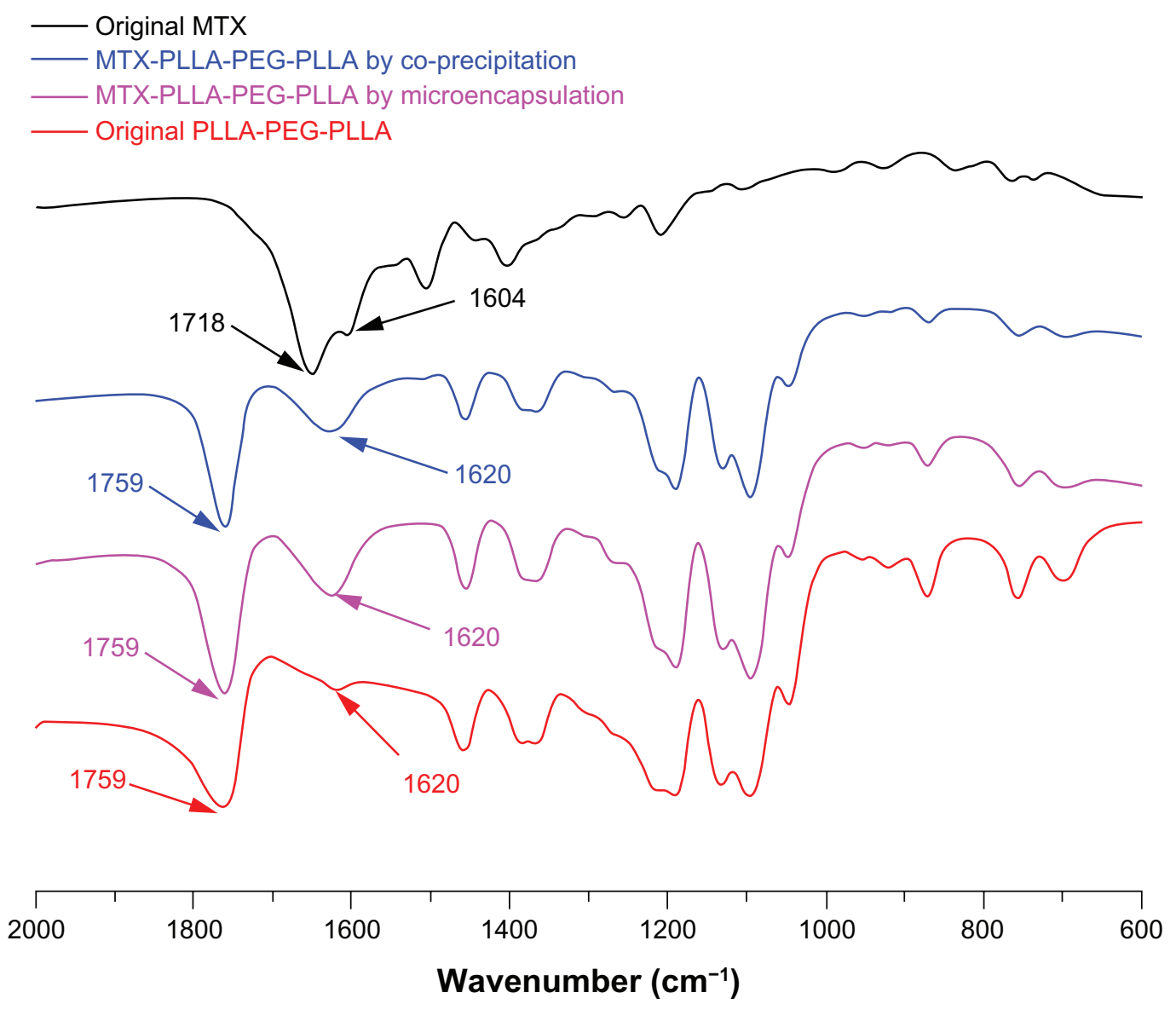

Figure 4 Fourier transform infrared spectra of methotrexate, PLLA-PEG-PLLA, and MTX-PLLA-PEG-PLLA microspheres from the microencapsulation process and the coprecipitation process.

Abbreviations: MTX, methotrexate; PLLA-PEG-PLLA, poly(L-lactide)-poly(ethylene glycol)-poly(L-lactide).

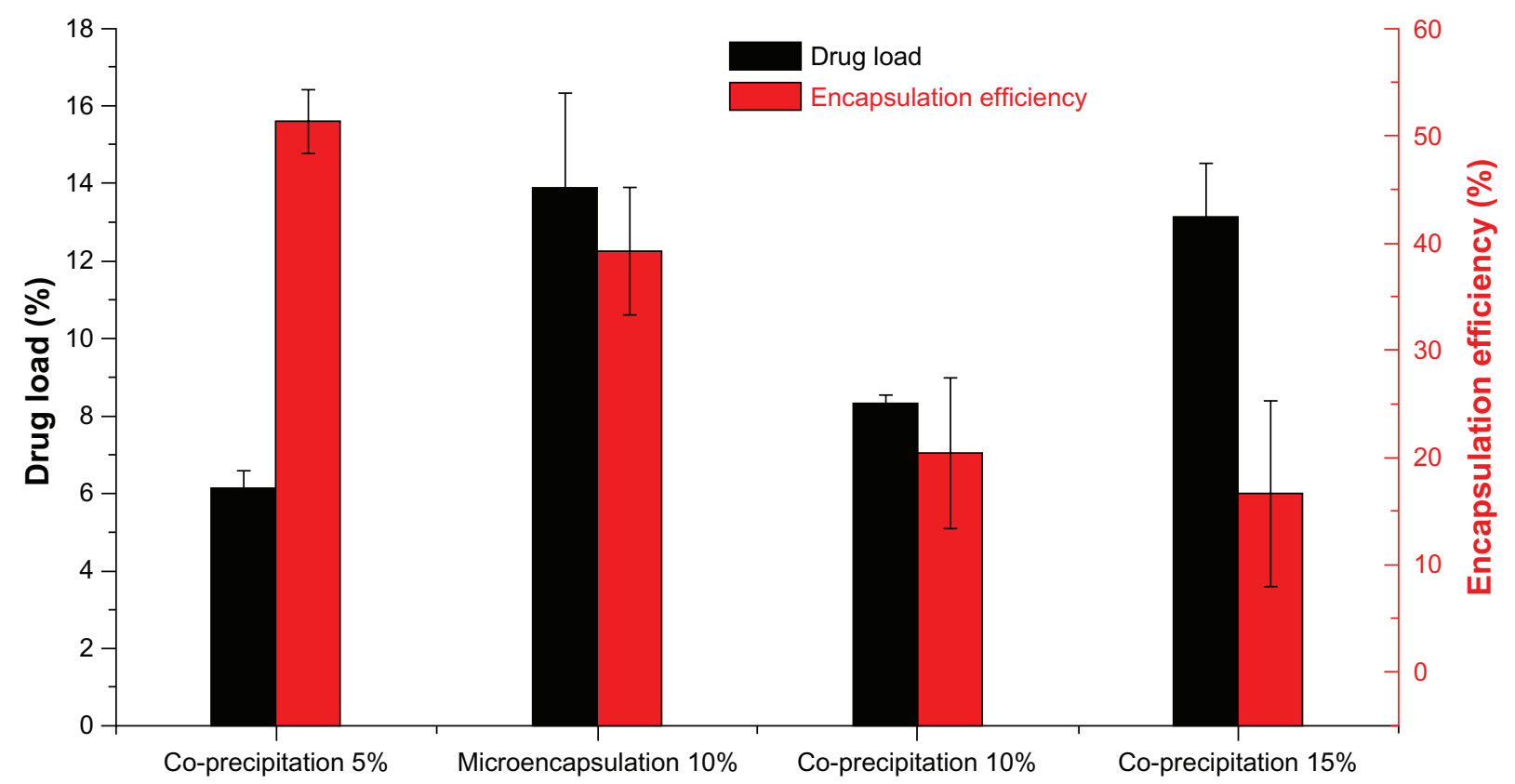

Figure 5 Drug loading and encapsulation efficiency of MTX-PLLA-PEG-PLLA microspheres from the microencapsulation process and the coprecipitation process, with different drug doses.

Abbreviation: MTX-PLLA-PEG-PLLA, methotrexate-poly(L-lactide)-poly(ethylene glycol)-poly(L-lactide). 
encapsulation efficiency decreased with the increase in drug dose. When drug doses were set at 5\%,10\%, and $15 \%$, the corresponding drug loading was $6.1 \% \pm 0.1 \%$, $8.3 \% \pm 0.4 \%$, and $13.1 \% \pm 1.4 \%$, together with an encapsulation efficiency of $51.3 \% \pm 3.0 \%, 20.4 \% \pm 6.3 \%$, and $16.6 \% \pm 8.0 \%$, respectively. When the drug dose was larger, more methotrexate would be precipitated at any given time point. Furthermore, the precipitation speed of methotrexate was lower than that of PLLA-PEG-PLLA in supercritical $\mathrm{CO}_{2}$, ie, the precipitated PLLA-PEG-PLLA acted as a crystal nucleus and in turn induced coprecipitation of methotrexate and PLLA-PEG-PLLA. These both contributed to the precipitation of more methotrexate on the surface of the preexisting MTX-PLLA-PEG-PLLA composite microspheres, generating microspheres with higher drug loading and lower encapsulation efficiency.

\section{Drug release profiles}

Methotrexate is a poorly water-soluble drug with a short half-life in vivo. An effective way to lengthen its half-life and enhance its therapeutic efficacy is to entrap it with biodegradable polymers to supply a sustained-release effect. Figure 6 illustrates the release profiles of MTX-PLLA-PEG-PLLA microspheres manufactured by the microencapsulation process and by the coprecipitation process using different drug doses. When the drug dose was set at $10 \%$ (w/w), in the first 30 minutes, $39.7 \%$ of the total methotrexate was released from the microspheres prepared by the microencapsulation process, while microspheres fabricated by the coprecipitation process released $42.3 \%$ of its methotrexate, both with an initial burst release. The methotrexate released rapidly at an early stage was unencapsulated or loosely adhered onto the microsphere surface and thus easily extracted, which again confirmed the result obtained by measurement of drug loading and encapsulation efficiency. After 30 hours, the cumulative percentage of the methotrexate released from the corresponding two kinds of microspheres went up to $86.0 \%$ and $89.8 \%$, respectively. In the following hours, there was a typical plateau period. At that time, the amount of drug remaining was rather limited, with sustained and slow drug release. When the plateau period was extended to 144 hours, the cumulative drug release percentage of the two kinds of composite microspheres rose to $91.7 \%$ and $94.1 \%$, respectively.

With regard to the coprecipitation process, samples with a drug dose of 5\% (w/w) released methotrexate more slowly and showed more desirable sustained release at the late stage, compared with the other two samples containing drug doses

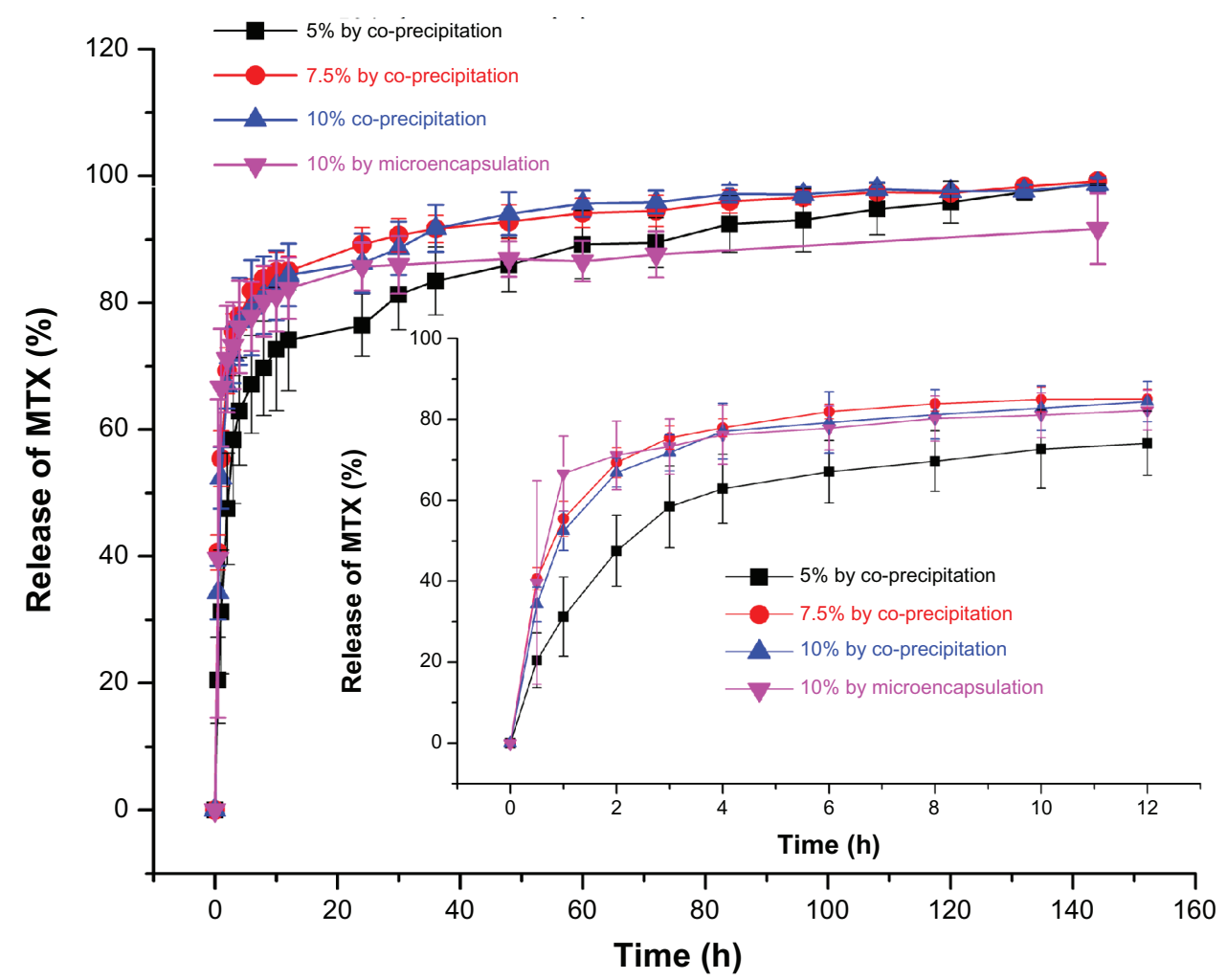

Figure 6 Drug release profiles of MTX-PLLA-PEG-PLLA microspheres from (A) the microencapsulation process and (B) the coprecipitation process with different drug doses. Abbreviation: MTX-PLLA-PEG-PLLA, methotrexate-poly(L-lactide)-poly(ethylene glycol)-poly(L-lactide). 
of $10 \%(\mathrm{w} / \mathrm{w})$ and $15 \%(\mathrm{w} / \mathrm{w})$, between which there was no significant difference.

It can also be seen in Figure 6 that drug release within 12 hours is a first-order process (correlation coefficient 0.99011, standard deviation 1.4709) for the MTX-PLLAPEG-PLLA microspheres prepared by the microencapsulation process, ${ }^{31}$ and after 12 hours, methotrexate started to be released in a zero-order kinetics manner, with the correlation coefficient and standard deviation being 0.97960 and 0.3393 , respectively. This result demonstrates that drug release from MTX-PLLA-PEG-PLLA microspheres prepared by the microencapsulation process was dominated by drug diffusion for the first 12 hours and mainly by polymer erosion thereafter, which can be explained by the fact that relatively large quantities of methotrexate nanoparticles had been encapsulated by PLLA-PEG-PLLA.

Methotrexate is an antimetabolite and antifolate drug than can prevent formation of thymine within the cell by inhibiting dihydrofolate reductase, rendering it incapable of DNA replication. Methotrexate has been widely used as a chemotherapy agent for cancer and arthritis. As shown in Figure 6, MTX-PLLA-PEG-PLLA prepared by the microencapsulation process can provide desirable sustained in vitro release of methotrexate, which can be used in the treatment of ectopic pregnancy ${ }^{32}$ and leukemia ${ }^{33}$ by intravenous injection, in breast cancer ${ }^{34}$ by pulmonary delivery, and in psoriasis ${ }^{35}$ and rheumatoid arthritis ${ }^{36}$ by percutaneous administration.

First-order kinetics are obeyed for drug release from MTX-PLLA-PEG-PLLA microspheres prepared by the coprecipitation process (the corresponding correlation coefficients and standard deviations for different drug doses are shown in Table 2). This indicates that drug release from MTX-PLLA-PEG-PLLA microspheres prepared by the coprecipitation process is dominated by drug diffusion, confirming that most methotrexate nanoparticles are absorbed or combined into the microsphere surface. Overall, cumulative release of MTX-PLLA-PEG-PLLA microspheres prepared

Table 2 Correlation coefficient and standard deviation obtained from the first-order release kinetics fitting of drug release profiles of MTX-PLLA-PEG-PLLA microspheres prepared by the coprecipitation process with different drug doses

\begin{tabular}{lll}
\hline Drug dose & $\mathbf{R}$ & SD \\
\hline $5 \%$ & 0.96490 & 1.5354 \\
$10 \%$ & 0.97409 & 1.5243 \\
$15 \%$ & 0.95187 & 1.9383 \\
\hline
\end{tabular}

Abbreviations: $R$, correlation coefficient; SD, standard deviation; MTX-PLLAPEG-PLLA, methotrexate, poly(L-lactide)-poly(ethylene glycol)-poly(L-lactide). by the microencapsulation process appeared to be more smooth and slower than that of microspheres prepared by the coprecipitation process.

\section{Conclusion}

With the addition of an injector in the suspension delivery system, SpEDS was effective in preventing damage to the HPLC pump and obstruction of the one-way valve, and was shown to be a successfully modified version of the original SEDS procedure. By suspending methotrexate nanoparticles in an PLLA-PEG-PLLA solution containing dichloromethane, composite microspheres were manufactured by a microencapsulation process, with higher drug loading and encapsulation efficiency and more desirable sustained release than those produced by the coprecipitation process. This research demonstrates that microencapsulation of drugs with a biodegradable polymer via SpEDS affords great potential for designing a controlled-release drug system.

\section{Acknowledgments}

The authors gratefully acknowledge the financial support of the Natural Science Foundation of Fujian Province (2010 J05027, 2011J01223) and the National Natural Science Foundation of China (51103049, 81171471, 31170939).

\section{Disclosure}

The authors report no conflicts of interest in this work.

\section{References}

1. Yuan WE, Liu ZG. Controlled-release and preserved bioactivity of proteins from (self-assembled) core-shell double-walled microspheres. Int J Nanomedicine. 2012;7:257-270.

2. Alli SMA. Formulation and evaluation of Bacillus coagulans-loaded hypromellose mucoadhesive microspheres. Int J Nanomedicine. 2011;6:619-629.

3. Karavelidis V, Karavas E, Giliopoulos D, et al. Evaluating the effects of crystallinity in new biocompatible polyester nanocarriers on drug release behavior. Int J Nanomedicine. 2011;6:3021-3032.

4. Sahana B, Santra K, Basu S, et al. Development of biodegradable polymer based tamoxifen citrate loaded nanoparticles and effect of some manufacturing process parameters on them: a physicochemical and in-vitro evaluation. Int J Nanomedicine. 2010;5:621-630.

5. Pouponneau P, Leroux JC, Soulez G, Gaboury L, Martel S. Co-encapsulation of magnetic nanoparticles and doxorubicin into biodegradable microcarriers for deep tissue targeting by vascular MRI navigation. Biomaterials. 2011;32(13):3481-3486.

6. Yin WS, Yates MZ. Encapsulation and sustained release from biodegradable microcapsules made by emulsification/freeze drying and spray/freeze drying. J Colloid Interface Sci. 2009;336(1):155-161.

7. Zvonar A, Berginc K, Kristl A, Gašperlin M. Microencapsulation of self-microemulsifying system: improving solubility and permeability of furosemide. Int J Pharm. 2010;388(1-2):151-158.

8. Fu S, Zhang G, Ding L, Tian A, Wang C. Encapsulation of disperse dye by phase separation technique using poly(styrene-maleic acid). $J$ Appl Polym Sci. 2011;120(6):3581-3586. 
9. Xi J, Zhou L, Fei Y. Preparation of chondroitin sulfate nanocapsules for use as carriers by the interfacial polymerization method. Int J Biol Macromol. 2012;50(1):157-163.

10. Beck-Broichsitter M, Schweiger C, Schmehl T, Gessler T, Seeger W, Kissel T. Characterization of novel spray-dried polymeric particles for controlled pulmonary drug delivery. J Control Release. 2012;158(2): 329-335.

11. Wang T, Wang N, Wang T, Sun W, Li T. Preparation of submicron liposomes exhibiting efficient entrapment of drugs by freeze-drying water-in-oil emulsions. Chem Phys Lipids. 2011;164(2):151-157.

12. Jelvehgari M, Barar J, Valizadeh H, Shadrou S, Nokhodchi A. Formulation, characterization and in vitro evaluation of theophyllineloaded Eudragit RS 100 microspheres prepared by an emulsion-solvent diffusion/evaporation technique. Pharm Dev Technol. 2011;16(6): 637-644.

13. He WZ, Jiang ZH, Suo QL, Li GM. Mechanism of dispersing an active component into a polymeric carrier by the SEDS-PA process. J Mater Sci. 2010;45(2):467-474

14. Du Z, Guan YX, Yao SJ, Zhu ZQ. Supercritical fluid assisted atomization introduced by an enhanced mixer for micronization of lysozyme: particle morphology, size and protein stability. Int J Pharm. 2011;421(2):258-268.

15. Zahran F, Morère J, Cabañas A, Renuncio JAR, Pando C. Role of excess molar enthalpies in supercritical antisolvent micronizations using dimethylsulfoxide as the polar solvent. J Supercrit Fluid. 2011;60:45-50.

16. Kim MS, Kim JS, Park HJ, Cho WK, Cha KH, Hwang SJ. Enhanced bioavailability of sirolimus via preparation of solid dispersion nanoparticles using a supercritical antisolvent process. Int J Nanomedicine. 2011;6:2997-3009.

17. Kalani M, Yunus R, Abdullah N. Optimizing supercritical antisolvent process parameters to minimize the particle size of paracetamol nanoencapsulated in L-polylactide. Int J Nanomedicine. 2011;6:1101-1105.

18. Kalani M, Yunus R. Application of supercritical antisolvent method in drug encapsulation: a review. Int J Nanomedicine. 2011;6:1429-1442.

19. Cocero MJ, Martín Á, Mattea F, Varona S. Encapsulation and coprecipitation processes with supercritical fluids: fundamentals and applications. J Supercrit Fluid. 2009;47(3):546-555.

20. Kompella UB, Koushik K. Preparation of drug delivery systems using supercritical fluid technology. Crit Rev Ther Drug Carr Syst. 2001;18(2):173-199.

21. Chen AZ, LiY, Chau FT, et al. Microencapsulation of puerarin nanoparticles by poly(l-lactide) in a supercritical $\mathrm{CO}_{2}$ process. Acta Biomater. 2009;5(8):2913-2919.

22. Wang YL, Wang YP, Yang J, Pfeffer R, Dave R, Michniak B. The application of a supercritical antisolvent process for sustained drug delivery. Powder Technol. 2006;164(2):94-102.
23. Tabernero A, Martín del Valle EM, Galán MA. Precipitation of tretinoin and acetaminophen with solution enhanced dispersion by supercritical fluids (SEDS). Role of phase equilibria to optimize particle diameter. Powder Technol. 2012;217:177-188.

24. Chen AZ, Zhao Z, Wang SB, Li Y, Zhao C, Liu YG. A continuous RESS process to prepare PLA-PEG-PLA microparticles. J Supercrit Fluid. 2011;59:92-97.

25. Li Y, Yang DJ, Chen SL, Chen SB, Chan ASC. Comparative physicochemical characterization of phospholipid complex of puerarin formulated by conventional and supercritical methods. Pharm Res. 2008;25(3):563-577.

26. Jung J, Perrut M. Particle design using supercritical fluids: literature and patent survey. J Supercrit Fluid. 2001;20(3):179-219.

27. Chen AZ, Li Y, Chen D, Hu JY. Development of core-shell microcapsules by a novel supercritical $\mathrm{CO}_{2}$ process. J Mater Sci Mater Med. 2009;20(3):751-758.

28. Pugi A, Benemei S, Vietri M, et al. Anaphylaxis during the first course of high-dose methotrexate: a case report and literature review. J Clin Pharm Ther. 2012;37(2):245-248.

29. Chen AZ, Li L, Wang SB, et al. Nanonization of methotrexate by solution-enhanced dispersion by supercritical $\mathrm{CO}_{2}$. J Supercrit Fluid. 2012;67:7-13

30. Torino E, De Marco I, Reverchon E. Organic nanoparticles recovery in supercritical antisolvent precipitation. J Supercrit Fluid. 2010;55(1): 300-306.

31. Wang SB, Chen AZ, Weng LJ, Chen MY, Xie XL. Effect of drugloading methods on drug load, encapsulation efficiency and release properties of alginate/poly-L-arginine/chitosan ternary complex microcapsules. Macromol Biosci. 2004;4(1):27-30.

32. Sagiv R, Debby A, Feit H, Cohen-Sacher B, Keidar R, Golan A. The optimal cutoff serum level of human chorionic gonadotropin for efficacy of methotrexate treatment in women with extrauterine pregnancy. Int J Gynecol Obstet. 2012;116(2):101-104.

33. Grossi O, Horeau-Langlard D, Agard C, et al. Low-dose methotrexate in PAH related to T-cell large granular lymphocyte leukaemia. Eur Respir J. 2012;39(2):493-494.

34. Buckman R. Metronomic cyclophosphamide and methotrexate for breast cancer: enhanced efficacy by adding dalteparin and low-dose prednisone? Breast. 2011;20(5):483-484

35. Srisuk P, Thongnopnua P, Raktanonchai U, Kanokpanont S. Physico-chemical characteristics of methotrexate-entrapped oleic acidcontaining deformable liposomes for in vitro transepidermal delivery targeting psoriasis treatment. Int J Pharm. 2012;427(2):426-434.

36. Lee SW, Kim JH, Park MC, et al. Alleviation of rheumatoid arthritis by cell-transducible methotrexate upon transcutaneous delivery. Biomaterials. 2012;33(5):1563-1572.
International Journal of Nanomedicine

\section{Publish your work in this journal}

The International Journal of Nanomedicine is an international, peerreviewed journal focusing on the application of nanotechnology in diagnostics, therapeutics, and drug delivery systems throughout the biomedical field. This journal is indexed on PubMed Central, MedLine, CAS, SciSearch ${ }^{\circledR}$, Current Contents ${ }^{\circledR} /$ Clinical Medicine,

\section{Dovepress}

Journal Citation Reports/Science Edition, EMBase, Scopus and the Elsevier Bibliographic databases. The manuscript management system is completely online and includes a very quick and fair peer-review system, which is all easy to use. Visit http://www.dovepress.com/ testimonials.php to read real quotes from published authors. 